\title{
Heterosis in single cross inter and intra-specific hybrids of Desi cotton (Gossipium arboreum and G. herbaceaum) for their seed cotton yield, fibre quality and seed oil content
}

\author{
Rakesh Choudhary $^{1 *}$, B. G. Solanki ${ }^{2}$, Navin Chander Gahtyari ${ }^{1}$, Tapas Paul ${ }^{3}$ and D. M. Patel ${ }^{2}$ \\ ${ }^{1}$ Department of Genetics and Plant Breeding, G. B. Pant University of Agriculture \& Technology, Pantnagar (UK), INDIA \\ ${ }^{2}$ Main Cotton Research Station, Navsari Agricultural University, Surat- 395007 (Gujarat), INDIA \\ ${ }^{3}$ ICAR Research Complex for NEH Region, Tripura Centre, INDIA \\ *Corresponding author. E-mail:- agrico.rakesh@gmail.com
}

Received: November 29, 2015; Revised received: April 30, 2016; Accepted: July 25, 2016

\begin{abstract}
The present investigation was carried out to assess the expression of per se performance and heterotic effect for fibre quality and seed oil content besides seed cotton yield, studied involving ten desi cotton (Gossipium arboreum and $G$. herbaceaum) genotypes and their 45 cross combinations in half diallel analysis. $F_{-1}$ hybrids GBhv282 x G 27 (67.36\%), GBhv- 287 x 824 (58.14\%), GBhv- 282 x GAM- 173 (35.00\%), GBhv- 286 × G 27 (20.50\%), and GBhv- $283 \times 824(18.75 \%)$ recorded highest per se performance and significant positive standard heterosis while the maximum heterobeltiosis for seed cotton yield per plant was exhibited by the hybrid GBhv- $287 \times 824$ (155.60 \%) followed by GBhv- 282 x G 27 (151.29\%) and GBhv- 282 x GAM- 173 (130.30\%). Similar trend of heterosis for numbers of boll per plant were observed in above hybrids. For fibre quality traits none of the cross showed consistent high performance for all the characters. Cross GBhv- $283 \times 824$ was exhibited high standard heterosis for $2.5 \%$ span length, fibre strength, fibre elongation percentage as well as for short fibre index (SFI) while cross GBhv$286 \times 824$ were promising for 2.5 per cent span length, fibre strength and fibre fineness. In case of oil content intraarboreum crosses resulted as better crosses and among them cross combination 824 x GAM- 173 was best. Desi cotton hybrids are having lower fibre quality and yield. So, improvement for yield and fibre quality of diploid native varieties through heterosis breeding provided better hybrids for rainfed farming.
\end{abstract}

Keywords: Diploid, Heterobeltiosis, Heterosis Breeding, Quantitative, Useful heterosis

\section{INTRODUCTION}

Cotton is a principle fiber, crop and plays a vital role as a cash crop in commerce of many countries. Cotton production, processing and trade in cotton goods provide employment to about 60 million people in India. It provides fibre for textile industry, cellulose from its lint, oil and protein rich meal from its seed (Ashok Kumar and Ravikesavan, 2011). The cotton seed, a by-product, is an important source of edible oil. Cotton seed is the second largest source of vegetable oil in the world. In India, secondary source of edible oil production is 19.90 lakh tons. Cotton seed oil is generally considered as healthy vegetable oil. The physical and chemical properties of cotton seed oil are well defined and in natural form have no cholesterol and are very low in trans fatty acid and hence termed as "Heart oil". When India got Independence, 97 per cent of the total cultivation was of Native diploid "Desi" cottons (varieties of Gossypium arboreum L. and G. herbaceum L). Gradually, desi varieties disappeared from the fields of Indian farmers with hybrids and $B t$ cotton replacing them in a very short time. This year, the cultivation has reached an abysmal low of three per cent. The desi varieties have ceded ground to the American varieties and now the situation is exactly the reverse, compared to that of 1947. Indian desi cotton is famous for its medicinal use due its absorbant ability and apart from surgical quality it is used general uses like textile, jeans, tea-coffee filters, fishing nets etc., whose demand is growing and the market price is very high. Desi varieties have resistance to pest and disease and can be grown with little rain and are suitable for dryland and mixed farming.

Cotton breeders are trying to develop cotton varieties; those well adapt to poor environmental conditions and produce higher yields and better fiber quality along with increased tolerance to complexes of diseases and insect pests. Very less number of desi cotton hybrids are released in India and the development of new desi cotton hybrid with high yield and fibre quality is necessary for Indian cotton farming.

Heterosis is a performance of $F_{1} / F_{2}$ genotypic combinations and is useful in determining the most appropriate parents for specific traits (Khan et al., 2010). Development of hybrid varieties is considered to be the quickest breeding method for exploiting the heterosis to improve yield potential of crop plants. Heterosis has 
substantially remained as one of the significant developments in cotton breeding programs. The yield increase through intra- and inter-specific heterosis over the better parent or best commercial cultivar (useful heterosis) has been documented (Wei et al., 2002; Yuan et al., 2002; Zhang et al., 2002; Khan et al. 2007a).

Several studies have been reported on seeds traits, but little work has been reported on the genetics and heterosis of cottonseed oil percentage in cotton breeding. A few reports in the literature (Khan et al., 2007b; Ganapathy and Nadarajan, 2008; Sharma et al., 2009; Khan et al., 2010) have determined that cotton genotypes differ in oil percentage.

Seed quality generally increases with high level of seed oil but had inverse relationship with seed cotton yield. For a simultaneous selection of both quality and yield, knowledge of inter relationship between the components of quality and those of yield is a prerequisite. In cotton, heterosis studies for seed cotton yield and other fiber properties are many, But to know the nature and extent of heterosis for oil content with seed cotton yield and other traits are limited.

Therefore, the present investigation objective was to estimate genetic variation for mean performance of parents and their hybrids and to estimate the effects of heterosis in $F_{1}$ cross combinations, to obtain information about heterotic potential as to develop hybrid with improved yield, fibre quality along with high seed oil content in diploid cotton (Gossipium arboreum and Gossipium herbaceaum).

\section{MATERIALS AND METHODS}

Genetic material: The material used in present study consisted of ten well adapted parental line of diploid cotton from diverse geographic origin, their $45 \mathrm{~F}_{1}$ cross combination and one standard check (SC) G. Cot DH7. Ten parents included five lines of Gossypium herbaceum cotton viz. GShv- 273/07, GBhv- 282, GBhv283, GBhv- 286 and GBhv- 287 and five lines of $G$. arboreum cotton viz. 824, G 27, GAM- 141, GAM165 and GAM- 173 (Table 1).

Experimental design and field procedures: The desi cotton cultivars and $\mathrm{F}_{1}$ cross combinations were evaluated in randomized block design (RBD) with three replications at Main Cotton Research Station, Surat (Gujarat) India. Each genotype was grown in a row of $4 \mathrm{~m}$ length adopting a spacing of $120 \mathrm{~cm}$ between rows and $40 \mathrm{~cm}$ between the plants, so as to have 10 plants per row. Parents were crossed in a half-diallel (excluding reciprocals) fashion during kharif 2011-12 to generate a total 45 hybrids. The conventional hand emasculation and pollination method developed by Dock and Moll (1934) was followed and crossed bolls collected separately and ginned to obtain $F_{1}$ seeds. During kharif 2012-13, a set of 56 entries (10 parents and $45 \mathrm{~F}_{1}$ 's and one standard check) were raised with three replications.

Sampling trait measurements and analysis:: In each genotypes and their cross combinations, data were recorded on five randomly selected plants per replication for eleven characters namely, seed cotton yield per plant (g), numbers of bolls per plant, boll weight (gm), $2.5 \%$ span length $(\mathrm{mm})$, fibre strength $\left(\mathrm{g} \mathrm{tex}^{-1}\right)$, fibre fineness $(\mathrm{mv})$, fibre elongation percentage $(\%)$, fibre maturity coefficient, short fibre index (SFI), fibre uniformity ratio $(\%)$ and seed oil content. Fibre quality parameters were analyzed using high volume instrument (HVI) (Premier Evolvics Pvt. Ltd., Coimbatore) in ideal condition (R.H. $65 \pm 2 \%$ and temperature $27 \pm 2^{\circ} \mathrm{C}$ ) at CIRCOT, Regional Station, Surat. The oil content was determined by Nuclear Magnetic Resonance (NMR) technique.

Heterosis was estimated over the better parent (BP) as per the standard procedure of Meredith and Bridge (1972) and useful heterosis as per standard method suggested by Rai (1978) over the standard check (SC). Data were analyzed using standard statistical software (INDOSTAT) to work out the magnitude of heterosis expressed by the hybrids under evaluation overbetter parent and standard check.

\section{RESULTS AND DISCUSSION}

Evaluation of mean performance and heterotic effect is essential to know whether new hybrids are suitable for direct exploitation or can be used to isolate transgressive segregants from subsequent generation to develop a new variety. The direct exploitation of hybrids for commercial production mainly depends upon the nature and extent of heterosis. The magnitude of heterosis of all cross combinations was worked out for all the characters over better parent and standard check. (Table 3)

Analysis of variance: The analysis of variance of RBD revealed that mean sum of squares due to differences among the genotypes was significant for all the characters studied, which indicates existence of substantial variability in the in the breeding material to be exploited in the study (Table 2). The variance due to parents was highly significant for all the characters. The mean square due to hybrids was significant for all the characters except fibre maturity coefficient. Significance of variance in parents versus hybrids interaction provided adequacy for comparing the heterotic expression for all the characters except for fibre uniformity ratio and oil content.

Genetic variability in $F_{1}$ hybrids and their parents: The mean expression of 11 characters was recorded on all 10 genotypes and 45 hybrids. All the genotypes and $F_{1}$ hybrids differ significantly for all the characters. The mean performance of parents are presented in Table-1 and the estimates of mean performance of hybrids are presented in Table-3 along with magnitude of heterosis over better parent (Heterobeltiosis) and standard check 
Rakesh Choudhary et al. / J. Appl. \& Nat. Sci. 8 (3): 1356 - 1365 (2016)

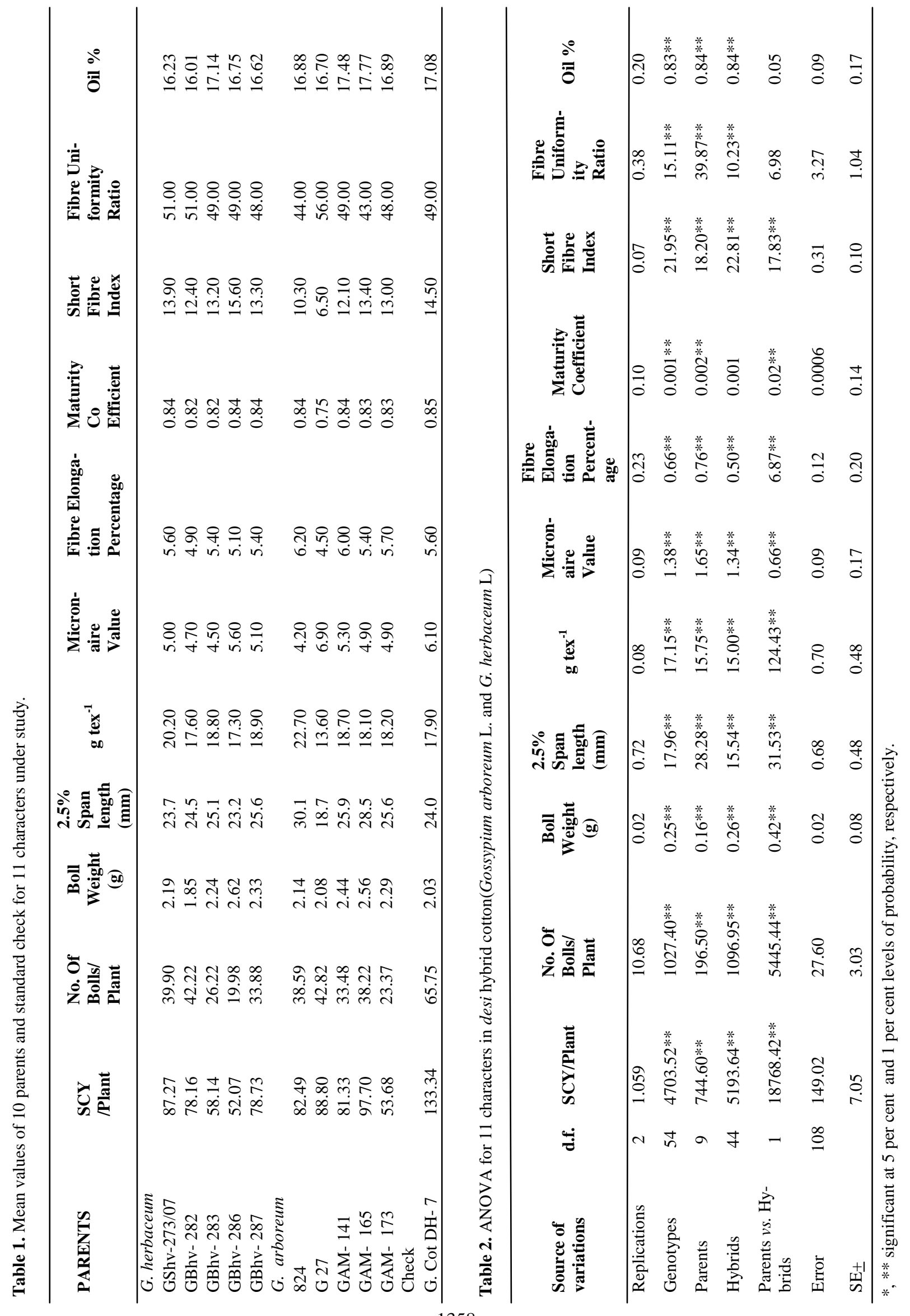


Rakesh Choudhary et al. / J. Appl. \& Nat. Sci. 8 (3): 1356 - 1365 (2016)

Table 3. Per se performance and heterosis over better parent (BP) and standard check (SC) for 11 different traits regarding to yield, fibre quality and oil content of desi cotton (Gossypium arboreum L. and G. herbaceum L.) hybrids.

\begin{tabular}{|c|c|c|c|c|c|c|c|c|c|c|}
\hline \multirow{2}{*}{$\begin{array}{l}\text { Sr. } \\
\text { No. }\end{array}$} & \multirow{2}{*}{ Crosses } & \multicolumn{3}{|c|}{ Seed cotton yield/ plant (g) } & \multicolumn{3}{|c|}{ No. of Bolls/ plant } & \multicolumn{3}{|c|}{ Boll Weight (g) } \\
\hline & & Mean & $\mathbf{B P}$ & SC & Mean & BP & SC & Mean & $\overline{\mathbf{B P}}$ & $\mathbf{S C}$ \\
\hline 1 & GShv- 273/07 x GBhv -282 & 90.62 & 3.84 & $-32.03 * *$ & 39.11 & -7.38 & $-40.52 * *$ & 2.31 & 5.48 & $13.79^{*}$ \\
\hline 2 & GShv- 273/07 x GBhv- 283 & 66.83 & $-23.42 *$ & $-49.88 * *$ & 29.69 & $-25.60 *$ & $-54.85^{* *}$ & 2.29 & 2.23 & $12.81^{*}$ \\
\hline 3 & GShv- 273/07 x GBhv- 286 & 69.81 & -20.01 & $-47.64 * *$ & 30.82 & $-22.76^{*}$ & $-53.13 * *$ & 2.26 & $13.74 * *$ & $11.33^{*}$ \\
\hline 4 & GShv- 273/07 x GBhv- 287 & 74.88 & -14.19 & $-43.84 * *$ & 26.73 & $-33.02 * *$ & $-59.35 * *$ & 2.81 & $20.60 * *$ & $38.42 * *$ \\
\hline 5 & GShv- 273/07 x 824 & 151.28 & $73.35^{* *}$ & 13.46 & 68.60 & $71.92 * *$ & 4.33 & 2.20 & 0.46 & 8.37 \\
\hline 6 & GShv- 273/07 x G 27 & 136.76 & $54.00^{* *}$ & 2.57 & 71.70 & $67.46^{* *}$ & 9.05 & 1.91 & $-12.79 *$ & -5.91 \\
\hline 7 & GShv- 273/07 x GAM- 141 & 123.67 & $41.71 * *$ & -7.25 & 56.85 & $42.47 * *$ & $-13.54 *$ & 2.17 & $-11.07 *$ & 6.90 \\
\hline 8 & GShv- 273/07 x GAM- 165 & 96.00 & -1.73 & $-28.00 * *$ & 39.78 & -0.31 & $-39.50 * *$ & 2.42 & -5.47 & $19.21 * *$ \\
\hline 9 & GShv- 273/07 x GAM- 173 & 98.84 & 13.26 & $-25.87 * *$ & 44.05 & 10.39 & $-33.01 * *$ & 2.25 & -1.75 & $10.84 *$ \\
\hline 10 & GBhv- $282 \times$ xBhv- 283 & 59.46 & -23.93 & $-55.41 * *$ & 22.66 & $-46.34 * *$ & $-65.54 * *$ & 2.65 & $18.30^{* *}$ & $30.54 * *$ \\
\hline 11 & GBhv- 282 x GBhv- 286 & 76.78 & -1.77 & $-42.42 * *$ & 34.60 & -18.05 & $-47.38 * *$ & 2.23 & $-14.89 * *$ & 9.85 \\
\hline 12 & GBhv- 282 x GBhv- 287 & 85.87 & 9.08 & $-35.60 * *$ & 38.97 & -7.70 & $-40.72 * *$ & 2.20 & -5.58 & 8.37 \\
\hline 13 & GBhv- 282 x 824 & 75.66 & -8.28 & $-43.26 * *$ & 36.15 & -14.40 & $-45.03 * *$ & 2.09 & -2.34 & 2.96 \\
\hline 14 & GBhv- 282 x G 27 & 223.15 & $151.29 * *$ & $67.36^{* *}$ & 97.40 & $127.48^{* *}$ & $48.14 * *$ & 2.29 & 10.10 & $12.81^{*}$ \\
\hline 15 & GBhv- 282 x GAM- 141 & 152.33 & $87.30 * *$ & 14.25 & 62.00 & $46.85 * *$ & -5.70 & 2.47 & 1.23 & $21.67 * *$ \\
\hline 16 & GBhv- 282 x GAM- 165 & 117.18 & 19.95 & -12.12 & 44.73 & 5.94 & $-31.96 * *$ & 2.62 & 2.34 & $29.06^{* *}$ \\
\hline 17 & GBhv- 282 x GAM- 173 & 180.00 & $130.30 * *$ & $35.00 * *$ & 83.86 & $98.60 * *$ & $27.54 * *$ & 2.14 & -6.40 & 5.58 \\
\hline 18 & GBhv- 283 x GBhv- 286 & 95.00 & $63.40^{* *}$ & $-28.75 * *$ & 39.08 & $49.08 * *$ & $-40.56^{* *}$ & 2.42 & -7.63 & $19.21 * *$ \\
\hline 19 & GBhv- 283 x GBhv- 287 & 92.33 & 17.28 & $-30.75 * *$ & 42.68 & $25.97 *$ & $-35.09 * *$ & 2.17 & -6.87 & 6.90 \\
\hline 20 & GBhv- 283 x 824 & 158.33 & $91.93^{* *}$ & $18.75^{*}$ & 76.56 & $98.37 * *$ & $16.44 *$ & 2.07 & -7.59 & 1.97 \\
\hline 21 & GBhv- 283 x G 27 & 105.57 & 18.88 & $-20.82 * *$ & 54.56 & $27.42 * *$ & $-17.02 *$ & 1.94 & $-13.39 * *$ & -4.43 \\
\hline 22 & GBhv- 283 x GAM- 141 & 95.01 & 16.82 & $-28.74 * *$ & 34.29 & 2.43 & $-47.85^{* *}$ & 2.76 & $13.11 * *$ & $35.96^{* *}$ \\
\hline 23 & GBhv- 283 x GAM- 165 & 65.86 & $-32.59 * *$ & $-50.61 * *$ & 22.41 & $-41.36 * *$ & $-65.91 * *$ & 2.94 & $14.84 * *$ & $44.83 * *$ \\
\hline 24 & GBhv- 283 x GAM- 173 & 73.99 & 27.26 & $-44.51 * *$ & 38.27 & $45.99 * *$ & $-41.79 * *$ & 1.95 & $-14.85^{* *}$ & -3.94 \\
\hline 25 & GBhv- 286 x GBhv- 287 & 111.00 & $40.99 * *$ & $-16.75^{*}$ & 50.94 & $50.34 * *$ & $-22.53 * *$ & 2.18 & $-16.79 * *$ & 7.39 \\
\hline 26 & GBhv- 286 x 824 & 122.22 & $48.16^{* *}$ & -8.34 & 61.70 & $59.86^{* *}$ & -6.16 & 1.98 & $-24.43 * *$ & -2.46 \\
\hline 27 & GBhv- 286 x G 27 & 160.67 & $80.92 * *$ & $20.50 * *$ & 79.59 & $85.89 * *$ & $21.05 * *$ & 2.02 & $-23.03 * *$ & -0.66 \\
\hline 28 & GBhv- 286 x GAM- 141 & 89.60 & 10.16 & $-32.80 * *$ & 48.35 & $44.43 * *$ & $-26.47 * *$ & 1.86 & $-29.01 * *$ & -8.37 \\
\hline 29 & GBhv- 286 x GAM- 165 & 54.93 & $-43.77 * *$ & $-58.80 * *$ & 29.09 & $-23.88 *$ & $-55.75^{* *}$ & 1.89 & $-27.86 * *$ & -6.90 \\
\hline 30 & GBhv- 286 x GAM- 173 & 79.33 & $47.78^{*}$ & $-40.50 * *$ & 37.36 & $59.85^{* *}$ & $-43.17 * *$ & 2.13 & $-18.70 * *$ & 4.93 \\
\hline 31 & GBhv- 287 x 824 & 210.86 & $155.60^{* *}$ & $58.14 * *$ & 93.09 & $141.22 * *$ & $41.59 * *$ & 2.30 & -1.29 & $13.30^{*}$ \\
\hline 32 & GBhv- 287 x G 27 & 146.73 & $65.23 * *$ & 10.04 & 72.29 & $68.83 * *$ & 9.94 & 2.02 & $-13.30 * *$ & -0.49 \\
\hline 33 & GBhv- 287 x GAM- 141 & 150.57 & $85.12 * *$ & 12.92 & 72.73 & $114.68 * *$ & 10.62 & 2.07 & $-15.16^{* *}$ & 1.97 \\
\hline 34 & GBhv- 287 x GAM- 165 & 128.74 & $31.78 * *$ & -3.45 & 62.83 & $64.39 * *$ & -4.44 & 2.05 & $-19.92 * *$ & 0.99 \\
\hline 35 & GBhv- 287 x GAM- 173 & 134.63 & $71.01 * *$ & 0.97 & 68.84 & $103.18^{* *}$ & 4.69 & 1.96 & $-15.88 * *$ & -3.45 \\
\hline 36 & 824 x G 27 & 73.16 & -17.62 & $-45.13 * *$ & 35.85 & -16.27 & $-45.48 * *$ & 2.10 & -2.02 & 3.28 \\
\hline 37 & 824 x GAM- 141 & 73.00 & -11.51 & $-45.25 * *$ & 38.23 & -0.94 & $-41.86 * *$ & 1.90 & $-22.13 * *$ & -6.40 \\
\hline 38 & $824 \times$ x GAM- 165 & 81.64 & -16.43 & $-38.77 * *$ & 42.37 & 9.78 & $-35.56 * *$ & 1.93 & $-22.48 * *$ & -4.76 \\
\hline 39 & $824 \times$ x GAM- 173 & 54.50 & $-33.93 * *$ & $-59.13 * *$ & 32.53 & -15.71 & $-50.52 * *$ & 1.68 & $-26.64 * *$ & $-17.24 * *$ \\
\hline 40 & G 27 x GAM- 141 & 70.10 & -21.06 & $-47.43 * *$ & 39.52 & -7.72 & $-39.90 * *$ & 1.77 & $-27.46^{* *}$ & $-12.81 *$ \\
\hline 41 & G $27 \times$ GAM- 165 & 86.33 & -11.63 & $-35.25 * *$ & 45.90 & 7.21 & $-30.19 * *$ & 1.88 & $-26.56 * *$ & -7.39 \\
\hline 42 & G 27 x GAM- 173 & 51.16 & $-42.39 * *$ & $-61.63 * *$ & 31.50 & $-26.43 * *$ & $-52.09 * *$ & 1.62 & $-29.26^{* *}$ & $-20.20 * *$ \\
\hline 43 & GAM- 141 x GAM- 165 & 92.14 & -5.68 & $-30.90 * *$ & 50.87 & $33.10^{* *}$ & $-22.63 * *$ & 1.81 & $-29.30 * *$ & $-10.84 *$ \\
\hline 44 & GAM- 141 x GAM- 173 & 62.28 & -22.43 & $-53.29 * *$ & 36.06 & 7.73 & $-45.16^{* *}$ & 1.73 & $-29.10 * *$ & $-14.78^{* *}$ \\
\hline \multirow[t]{4}{*}{45} & GAM- 165 x GAM- 173 & 58.20 & $-40.43 * *$ & $-56.35 * *$ & 29.11 & $-23.84^{*}$ & $-55.73 * *$ & 1.99 & $-22.27 * *$ & -1.97 \\
\hline & S.E. $(d) \pm$ & & 9.97 & 9.97 & & 4.29 & 4.29 & & 0.11 & 0.11 \\
\hline & CD 0.05 & & 19.76 & 19.76 & & 8.50 & 8.50 & & 0.21 & 0.21 \\
\hline & $\mathrm{CD} 0.01$ & & 26.14 & 26.14 & & 11.25 & 11.25 & & 0.28 & 0.28 \\
\hline
\end{tabular}

$*$, ** Significant at $5 \%$ and $1 \%$ levels, respectively. 
Rakesh Choudhary et al. / J. Appl. \& Nat. Sci. 8 (3): 1356 - 1365 (2016)

Table 3 Contd.....

\begin{tabular}{|c|c|c|c|c|c|c|c|c|c|c|}
\hline \multirow{2}{*}{$\begin{array}{l}\text { Sr. } \\
\text { No. }\end{array}$} & \multirow{2}{*}{ Crosses } & \multicolumn{3}{|c|}{2.5 per cent span length } & \multicolumn{3}{|c|}{ Fibre strength $\left(\mathrm{g} \mathrm{tex}^{-1}\right)$} & \multicolumn{3}{|c|}{ Fibre fineness (mv) } \\
\hline & & Mean & BP & SC & Mean & BP & SC & Mean & $\mathbf{B P}$ & $\mathbf{S C}$ \\
\hline 1 & GShv- 273/07 x GBhv -282 & 25.9 & $5.85^{*}$ & $8.06^{* *}$ & 21.80 & $8.09^{*}$ & $21.97 * *$ & 4.60 & -2.13 & $-24.59^{* * *}$ \\
\hline 2 & GShv- 273/07 x GBhv- 283 & 25.1 & -0.13 & 4.58 & 20.30 & 0.66 & $13.59 * *$ & 4.80 & 7.41 & $-20.77 * *$ \\
\hline 3 & GShv- 273/07 x GBhv- 286 & 25.2 & $6.18^{*}$ & 5.00 & 22.90 & $13.53^{* *}$ & $28.12^{* *}$ & 4.40 & $-12.00^{*}$ & $-27.87 * *$ \\
\hline 4 & GShv- 273/07 x GBhv- 287 & 25.7 & 0.39 & $7.08 *$ & 20.40 & 1.16 & $14.15^{* *}$ & 4.80 & -4.00 & $-21.31^{* *}$ \\
\hline 5 & GShv- 273/07 x 824 & 27.3 & $-9.29 *$ & $13.89^{* *}$ & 23.40 & 3.23 & $30.91 * *$ & 4.50 & 7.94 & $-25.68 * *$ \\
\hline 6 & GShv- 273/07 x G 27 & 25.1 & 5.62 & 4.44 & 18.60 & $-7.76^{*}$ & 4.10 & 6.20 & $24.00^{* *}$ & 1.64 \\
\hline 7 & GShv- 273/07 x GAM- 141 & 26.6 & 2.70 & $10.83^{* *}$ & 20.80 & 3.14 & $16.39^{* *}$ & 5.00 & 0.00 & $-18.03^{* *}$ \\
\hline 8 & GShv- 273/07 x GAM- 165 & 28.2 & -1.05 & $17.50^{* *}$ & 21.90 & $8.58^{*}$ & $22.53 * *$ & 5.10 & 4.08 & $-16.39 * *$ \\
\hline 9 & GShv- 273/07 x GAM- 173 & 27.7 & $8.20 * *$ & $15.42^{* *}$ & 21.20 & 5.12 & $18.62 * *$ & 5.10 & 4.08 & $-16.39^{* *}$ \\
\hline 10 & GBhv- 282 x GBhv- 283 & 26.6 & $5.84^{*}$ & $10.83^{* *}$ & 21.00 & $11.50^{* *}$ & $17.32 * *$ & 4.60 & 1.48 & $-25.14 * *$ \\
\hline 11 & GBhv- 282 x GBhv- 286 & 25.3 & 3.27 & 5.42 & 18.30 & 3.78 & 2.23 & 5.10 & 9.22 & $-15.85^{* *}$ \\
\hline 12 & GBhv- 282 x GBhv- 287 & 24.8 & -2.99 & 3.47 & 20.50 & $8.27 *$ & $14.53^{* *}$ & 4.90 & 4.26 & $-19.67 * *$ \\
\hline 13 & GBhv- $282 \times 824$ & 24.8 & $-17.70 * *$ & 3.33 & 20.90 & -7.78 & $16.95^{* *}$ & 4.90 & $17.46^{* *}$ & $-19.13^{* *}$ \\
\hline 14 & GBhv- $282 \times$ x 27 & 25.6 & 4.49 & $6.67^{*}$ & 22.10 & $25.52^{* *}$ & $23.65^{* *}$ & 6.40 & $36.17^{* *}$ & 4.92 \\
\hline 15 & GBhv- 282 x GAM- 141 & 27.6 & $6.56^{*}$ & $15.00^{* *}$ & 22.50 & $19.93^{* *}$ & $25.51^{* *}$ & 5.60 & $19.15^{* *}$ & $-8.20 *$ \\
\hline 16 & GBhv- 282 x GAM- 165 & 28.5 & 0.00 & $18.75^{* *}$ & 21.20 & $17.10^{* *}$ & $18.62^{* *}$ & 5.10 & 7.80 & $-16.94 * *$ \\
\hline 17 & GBhv- 282 x GAM- 173 & 27.6 & $7.94 * *$ & $15.14 * *$ & 22.00 & $20.84^{* *}$ & $23.09^{* *}$ & 5.10 & 9.22 & $-15.85^{* *}$ \\
\hline 18 & GBhv- 283 x GBhv- 286 & 26.7 & $6.23^{*}$ & $11.25^{* *}$ & 19.80 & 5.13 & $10.61 * *$ & 5.20 & $15.56^{* *}$ & $-14.75^{* *}$ \\
\hline 19 & GBhv- 283 x GBhv- 287 & 28.8 & $12.50^{* *}$ & $20.00^{* *}$ & 21.80 & $15.32^{* *}$ & $21.97 * *$ & 5.50 & $22.22 * *$ & $-9.84 *$ \\
\hline 20 & GBhv- $283 \times 824$ & 30.3 & 0.55 & $26.25^{* *}$ & 24.10 & $6.17^{*}$ & $34.64 * *$ & 4.60 & 9.52 & $-24.59 * *$ \\
\hline 21 & GBhv- $283 \times$ G 27 & 23.8 & -5.31 & -0.83 & 19.20 & 1.95 & 7.26 & 6.50 & $44.44^{* *}$ & 6.56 \\
\hline 22 & GBhv- 283 x GAM- 141 & 26.5 & 2.19 & $10.28 * *$ & 20.30 & $7.79^{*}$ & $13.41^{* *}$ & 5.20 & $15.56^{* *}$ & $-14.75^{* *}$ \\
\hline 23 & GBhv- 283 x GAM- 165 & 24.8 & $-12.98 * *$ & 3.33 & 19.60 & 4.07 & $9.50^{*}$ & 5.00 & $11.11 *$ & $-18.03 * *$ \\
\hline 24 & GBhv- 283 x GAM- 173 & 27.7 & $8.20 * *$ & $15.42^{* *}$ & 23.00 & $22.12^{* *}$ & $28.49^{* *}$ & 5.20 & $15.56^{* *}$ & $-14.75^{* *}$ \\
\hline 25 & GBhv- 286 x GBhv- 287 & 30.1 & $17.58^{* *}$ & $25.42^{* *}$ & 20.80 & $9.86^{* *}$ & $16.20^{* *}$ & 5.50 & 7.84 & $-9.84 *$ \\
\hline 26 & GBhv- $286 \times 824$ & 32.0 & $6.19 * *$ & $33.33^{* *}$ & 23.70 & 4.41 & $32.40 * *$ & 4.30 & 2.38 & $-29.51 * *$ \\
\hline 27 & GBhv- 286 x G 27 & 23.3 & 0.43 & -2.92 & 19.60 & $13.49^{* *}$ & $9.68 *$ & 6.00 & 7.14 & -1.64 \\
\hline 28 & GBhv- 286 x GAM- 141 & 25.9 & 0.00 & $7.92 * *$ & 20.60 & $9.96^{* *}$ & $15.08^{* *}$ & 4.80 & $-9.43 *$ & $-21.31 * *$ \\
\hline 29 & GBhv- 286 x GAM- 165 & 24.3 & $-14.62 * *$ & 1.39 & 21.40 & 18.20 & $19.74 * *$ & 4.30 & $-12.24 *$ & $-29.51 * *$ \\
\hline 30 & GBhv- 286 x GAM- 173 & 24.2 & $-5.47 *$ & 0.83 & 18.50 & 1.46 & 3.35 & 6.10 & $24.49 * *$ & 0.00 \\
\hline 31 & GBhv- 287 x 824 & 29.1 & -3.43 & $21.25^{* *}$ & 22.50 & -0.88 & $25.70^{* *}$ & 4.90 & $15.87^{* *}$ & $-20.22 * *$ \\
\hline 32 & GBhv- $287 \times$ x G 27 & 24.7 & -3.52 & 2.92 & 21.40 & $13.03^{* *}$ & $19.55^{* *}$ & 5.50 & 7.84 & $-9.84 *$ \\
\hline 33 & GBhv- 287 x GAM- 141 & 27.4 & $5.79^{*}$ & $14.17^{* *}$ & 21.60 & $14.08^{* *}$ & $20.67 * *$ & 4.50 & $-11.11^{*}$ & $-25.68 * *$ \\
\hline 34 & GBhv- 287 x GAM- 165 & 26.8 & $-5.96 *$ & $11.67^{* *}$ & 21.60 & $14.26 * *$ & $20.86^{* *}$ & 5.50 & $11.56^{*}$ & $-10.38 * *$ \\
\hline 35 & GBhv- 287 x GAM- 173 & 28.0 & $9.37 * *$ & $16.67^{* *}$ & 23.00 & $21.65^{* *}$ & $28.68^{* *}$ & 4.80 & -2.04 & $-21.31 * *$ \\
\hline 36 & $824 \times$ G 27 & 30.4 & 1.00 & $26.81^{* *}$ & 22.00 & -2.94 & $23.09^{* *}$ & 3.80 & $-9.52 * *$ & $-37.70^{* *}$ \\
\hline 37 & 824 x GAM- 141 & 22.3 & $-26.00 * *$ & $-7.08 *$ & 15.90 & $29.81 * *$ & $-10.99 * *$ & 6.40 & $52.38 * *$ & 4.92 \\
\hline 38 & 824 x GAM- 165 & 21.8 & $-27.65 * *$ & $-9.17 * *$ & 14.70 & $-35.10^{* *}$ & $-17.69^{* *}$ & 6.30 & $50.00^{* *}$ & 3.28 \\
\hline 39 & 824 x GAM- 173 & 25.2 & $-16.37 * *$ & 5.00 & 20.30 & $-10.43^{* *}$ & $13.59^{* *}$ & 5.70 & $35.71^{* *}$ & -6.56 \\
\hline 40 & G $27 \times$ GAM- 141 & 24.8 & -4.25 & 3.33 & 17.50 & -6.41 & -2.05 & 6.10 & $15.72 * *$ & 0.55 \\
\hline 41 & G 27 x GAM- 165 & 24.0 & $-15.67 * *$ & 0.14 & 18.90 & 4.41 & 5.77 & 5.80 & $18.37 * *$ & -4.92 \\
\hline 42 & G $27 \times$ GAM- 173 & 21.1 & $-17.58 * *$ & $-12.08^{* * *}$ & 13.20 & $-27.61 * *$ & $-26.26^{* *}$ & 6.50 & $32.65^{* *}$ & 6.56 \\
\hline 43 & GAM- $141 \times$ GAM- 165 & 27.1 & $-4.91 *$ & $12.92^{* *}$ & 20.20 & $8.01^{*}$ & $13.04 * *$ & 5.90 & $20.41^{* *}$ & -3.28 \\
\hline 44 & GAM- $141 \times$ GAM- 173 & 26.2 & 1.29 & $9.31 * *$ & 23.40 & $25.09^{* *}$ & $30.91^{* *}$ & 5.40 & $10.20^{*}$ & $-11.48^{* *}$ \\
\hline \multirow[t]{4}{*}{45} & GAM- 165 x GAM- 173 & 25.4 & $-10.88 * *$ & $5.83^{*}$ & 21.40 & $17.55^{* *}$ & $19.74 * *$ & 5.70 & $16.33^{* *}$ & -6.56 \\
\hline & S.E. (d) \pm & & 0.67 & 0.67 & & 0.68 & 0.68 & & 0.24 & 0.24 \\
\hline & CD 0.05 & & 1.34 & 1.34 & & 1.35 & 1.35 & & 0.48 & 0.48 \\
\hline & CD 0.01 & & 1.77 & 1.77 & & 1.79 & 1.79 & & 0.63 & 0.63 \\
\hline
\end{tabular}

*, ** Significant at $5 \%$ and $1 \%$ levels, respectively. 
Rakesh Choudhary et al. / J. Appl. \& Nat. Sci. 8 (3): 1356 - 1365 (2016)

Table 3. Contd.....

\begin{tabular}{|c|c|c|c|c|c|c|c|c|c|c|}
\hline & \multirow{2}{*}{ Crosses } & \multicolumn{3}{|c|}{ Fibre elongation percentage } & \multicolumn{3}{|c|}{ Maturity coefficient } & \multicolumn{3}{|c|}{ Short Fibre Index (SFI) } \\
\hline & & Mean & BP & SC & Mean & $\mathbf{B P}$ & SC & Mean & $\mathbf{B P}$ & SC \\
\hline 1 & GShv- 273/07 x GBhv -282 & 5.70 & 1.79 & 1.79 & 0.84 & 0.00 & -1.18 & 10.40 & $-16.13 * *$ & $-28.28 * *$ \\
\hline 2 & GShv- 273/07 x GBhv- 283 & 5.50 & -1.79 & -1.79 & 0.84 & 0.00 & -1.18 & 10.60 & $-19.70 * *$ & $-26.90 * *$ \\
\hline 3 & GShv- 273/07 x GBhv- 286 & 5.60 & 0.00 & 0.00 & 0.84 & 0.00 & -1.18 & 12.30 & $-11.51 * *$ & $-15.17 * *$ \\
\hline 4 & GShv- 273/07 x GBhv- 287 & 5.50 & -1.79 & -1.79 & 0.84 & 0.00 & -1.18 & 9.50 & $-28.57 * *$ & $-34.48 * *$ \\
\hline 5 & GShv- 273/07 x 824 & 6.20 & 0.00 & $10.71 *$ & 0.85 & 1.19 & 0.00 & 9.40 & $-8.74 *$ & $-35.17 * *$ \\
\hline 6 & GShv- 273/07 x G 27 & 5.90 & 5.36 & 5.36 & 0.86 & 2.38 & 1.18 & 11.70 & $80.00 * *$ & $-19.31 * *$ \\
\hline 7 & GShv- 273/07 x GAM- 141 & 5.90 & -1.67 & 5.36 & 0.85 & 1.19 & 0.00 & 11.60 & -4.13 & $-20.00 * *$ \\
\hline 8 & GShv- 273/07 x GAM- 165 & 6.50 & $16.07 * *$ & $16.07 * *$ & 0.86 & 2.38 & 1.18 & 8.00 & $-40.30 * *$ & $-44.83 * *$ \\
\hline 9 & GShv- 273/07 x GAM- 173 & 6.20 & 8.77 & $10.71 *$ & 0.85 & 1.19 & 0.00 & 9.90 & $-23.85 * *$ & $-31.72 * *$ \\
\hline 10 & GBhv- 282 x GBhv- 283 & 5.90 & 9.26 & 5.36 & 0.84 & 2.44 & -1.18 & 10.60 & $-14.52 * *$ & $-26.90 * *$ \\
\hline 11 & GBhv- 282 x GBhv- 286 & 5.60 & 9.80 & 0.00 & 0.83 & -1.19 & -2.35 & 11.00 & $-11.29 * *$ & $-24.14 * *$ \\
\hline 12 & GBhv- 282 x GBhv- 287 & 5.60 & 3.70 & 0.00 & 0.84 & 0.00 & -1.18 & 14.00 & $12.90 * *$ & -3.45 \\
\hline 13 & GBhv- 282 x 824 & 5.80 & -6.45 & 3.57 & 0.85 & 1.19 & 0.00 & 12.50 & $21.36^{* *}$ & $-13.79 * *$ \\
\hline 14 & GBhv- 282 x G 27 & 6.60 & $34.69 * *$ & $17.86^{* *}$ & 0.88 & $7.32 * *$ & 3.53 & 12.00 & $84.62 * *$ & $-17.24 * *$ \\
\hline 15 & GBhv- 282 x GAM- 141 & 6.40 & 6.67 & $14.29 * *$ & 0.87 & 3.57 & 2.35 & 8.30 & $-31.40 * *$ & $-42.76 * *$ \\
\hline 16 & GBhv- 282 x GAM- 165 & 6.30 & $16.67 * *$ & $12.50 *$ & 0.85 & 2.41 & 0.00 & 8.80 & $-29.03 * *$ & $-39.31 * *$ \\
\hline 17 & GBhv- 282 x GAM- 173 & 6.10 & 7.02 & 8.93 & 0.86 & 3.61 & 1.18 & 9.20 & $-25.81 * *$ & $-36.55^{* *}$ \\
\hline 18 & GBhv- 283 x GBhv- 286 & 5.60 & 3.70 & 0.00 & 0.85 & 1.19 & 0.00 & 10.20 & $-22.73 * *$ & $-29.66^{* *}$ \\
\hline 19 & GBhv- 283 x GBhv- 287 & 6.40 & $18.52 * *$ & $14.29 * *$ & 0.87 & 3.57 & 2.35 & 8.90 & $-32.58 * *$ & $-38.62 * *$ \\
\hline 20 & GBhv- 283 x 824 & 6.50 & 4.84 & $16.07 * *$ & 0.86 & 2.38 & 1.18 & 7.00 & $-32.04 * *$ & $-51.72 * *$ \\
\hline 21 & GBhv- 283 x G 27 & 6.10 & $12.96 *$ & 8.93 & 0.87 & $6.10^{*}$ & 2.35 & 13.00 & $100.00 * *$ & $-10.34 * *$ \\
\hline 22 & GBhv- 283 x GAM- 141 & 5.60 & -6.67 & 0.00 & 0.85 & 1.19 & 0.00 & 11.10 & $-8.26^{*}$ & $-23.45^{* *}$ \\
\hline 23 & GBhv- 283 x GAM- 165 & 5.30 & -1.85 & -5.36 & 0.84 & 1.20 & -1.18 & 12.70 & -3.79 & $-12.41 * *$ \\
\hline 24 & GBhv- 283 x GAM- 173 & 6.40 & $12.28 *$ & $14.29 * *$ & 0.86 & 3.61 & 1.18 & 10.40 & $-20.00 * *$ & $-28.28 * *$ \\
\hline 25 & GBhv- 286 x GBhv- 287 & 6.20 & $14.81 * *$ & $10.71 *$ & 0.86 & 2.38 & 1.18 & 6.80 & $-48.87 * *$ & $-53.10 * *$ \\
\hline 26 & GBhv- 286 x 824 & 6.20 & 0.00 & $10.71^{*}$ & 0.84 & 0.00 & -1.18 & 7.20 & $-30.10^{* *}$ & $-50.34 * *$ \\
\hline 27 & GBhv- 286 x G 27 & 6.30 & $23.53^{* *}$ & $12.50^{*}$ & 0.86 & 2.38 & 1.18 & 13.60 & $109.23 * *$ & $-6.21 *$ \\
\hline 28 & GBhv- 286 x GAM- 141 & 5.90 & -1.67 & 5.36 & 0.84 & 0.00 & -1.18 & 12.90 & 6.61 & $-11.03 * *$ \\
\hline 29 & GBhv- 286 x GAM- 165 & 5.50 & 1.85 & -1.79 & 0.83 & -1.19 & -2.35 & 13.90 & 3.73 & -4.14 \\
\hline 30 & GBhv- 286 x GAM- 173 & 6.10 & 7.02 & 8.93 & 0.86 & 2.38 & 1.18 & 14.20 & $9.23 * *$ & -2.07 \\
\hline 31 & GBhv- 287 x 824 & 6.50 & 4.84 & $16.07 * *$ & 0.85 & 1.19 & 0.00 & 7.00 & $-32.04 * *$ & $-51.72 * *$ \\
\hline 32 & GBhv- 287 x G 27 & 6.30 & $16.67 * *$ & $12.50 *$ & 0.86 & 2.38 & 1.18 & 13.20 & 103.08 & $-8.97 * *$ \\
\hline 33 & GBhv- 287 x GAM- 141 & 6.20 & 3.33 & $10.71^{*}$ & 0.84 & 0.00 & -1.18 & 10.90 & $-9.92 * *$ & $-24.83 * *$ \\
\hline 34 & GBhv- 287 x GAM- 165 & 6.30 & $16.67 * *$ & $12.50 *$ & 0.86 & 2.38 & 1.18 & 10.00 & $-24.81 * *$ & $-31.03 * *$ \\
\hline 35 & GBhv- 287 x GAM- 173 & 6.40 & $12.28 *$ & $14.29 * *$ & 0.85 & 1.19 & 0.00 & 9.30 & $-28.46 * *$ & $-35.86 * *$ \\
\hline 36 & 824 x G 27 & 6.10 & -1.61 & 8.93 & 0.82 & -2.38 & -3.53 & 10.30 & $58.46^{* *}$ & $-28.97 * *$ \\
\hline 37 & 824 x GAM- 141 & 5.30 & $-14.52 * *$ & -5.36 & 0.84 & 0.00 & -1.18 & 15.90 & $54.37 * *$ & $9.66 * *$ \\
\hline 38 & 824 x GAM- 165 & 5.30 & $-14.52 * *$ & -5.36 & 0.83 & -1.19 & -2.35 & 16.70 & $62.14 * *$ & $15.17 * *$ \\
\hline 39 & 824 x GAM- 173 & 5.70 & -8.06 & 1.79 & 0.86 & 2.38 & 1.18 & 14.50 & $40.78^{* *}$ & 0.00 \\
\hline 40 & G 27 x GAM- 141 & 5.40 & $10.00 *$ & -3.57 & 0.85 & 1.19 & 0.00 & 14.30 & $120.00 * *$ & -1.38 \\
\hline 41 & G 27 x GAM- 165 & 5.70 & 5.56 & 1.79 & 0.85 & 2.41 & 0.00 & 15.60 & $140.00 * *$ & $7.59 *$ \\
\hline 42 & G 27 x GAM- 173 & 4.90 & $-14.04 * *$ & $-12.50 *$ & 0.83 & 0.00 & -2.35 & 19.20 & $195.38 * *$ & $32.41 * *$ \\
\hline 43 & GAM- 141 x GAM- 165 & 6.40 & 6.67 & $14.29 * *$ & 0.86 & 2.38 & 1.18 & 11.80 & -2.48 & $-18.62 * *$ \\
\hline 44 & GAM- 141 x GAM- 173 & 5.90 & -1.67 & 5.36 & 0.87 & 3.57 & 2.35 & 14.00 & $15.70 * *$ & -3.45 \\
\hline \multirow[t]{4}{*}{45} & GAM- 165 x GAM- 173 & 5.90 & 3.51 & 5.36 & 0.87 & $4.82 *$ & 2.35 & 13.90 & $6.92 *$ & -4.14 \\
\hline & S.E. (d) \pm & & 0.28 & 0.28 & & 0.02 & 0.02 & & 0.45 & 0.45 \\
\hline & CD 0.05 & & 0.56 & 0.56 & & 0.04 & 0.04 & & 0.90 & 0.90 \\
\hline & CD 0.01 & & 0.74 & 0.74 & & 0.05 & 0.05 & & 1.19 & 1.19 \\
\hline
\end{tabular}

*, ** Significant at $5 \%$ and $1 \%$ levels, respectively. 
Rakesh Choudhary et al. / J. Appl. \& Nat. Sci. 8 (3): 1356 - 1365 (2016)

Table 3. Contd.....

\begin{tabular}{|c|c|c|c|c|c|c|c|}
\hline \multirow{2}{*}{$\begin{array}{l}\text { Sr. } \\
\text { No }\end{array}$} & \multirow{2}{*}{ Crosses } & \multicolumn{3}{|c|}{ Fibre uniformity ratio } & \multicolumn{3}{|c|}{ Oil content $(\%)$} \\
\hline & & Mean & $\mathbf{B P}$ & SC & Mean & BP & SC \\
\hline 1 & GShv- 273/07 x GBhv -282 & 51.00 & 0.00 & 4.08 & 16.58 & 2.16 & $-2.93 *$ \\
\hline 2 & GShv- 273/07 x GBhv- 283 & 53.00 & 3.92 & $8.16^{* *}$ & 16.58 & $-3.27 *$ & $-2.93 *$ \\
\hline 3 & GShv- 273/07 x GBhv- 286 & 50.00 & -1.96 & 2.04 & 15.44 & $-7.82 * *$ & $-9.60 * *$ \\
\hline 4 & GShv- 273/07 x GBhv- 287 & 53.00 & 3.92 & $8.16^{* *}$ & 16.28 & -2.05 & $-4.68 * *$ \\
\hline 5 & GShv- 273/07 x 824 & 50.00 & -1.96 & 2.04 & 15.93 & $-5.63 * *$ & $-6.73 * *$ \\
\hline 6 & GShv- 273/07 x G 27 & 51.00 & $-8.93 * *$ & 4.08 & 16.40 & -1.80 & $-3.98 * *$ \\
\hline 7 & GShv- 273/07 x GAM- 141 & 48.00 & $-5.88^{*}$ & -2.04 & 16.97 & $-2.92 *$ & -0.64 \\
\hline 8 & GShv- 273/07 x GAM- 165 & 51.00 & 0.00 & 4.08 & 16.37 & $-7.88 * *$ & $-4.16^{* *}$ \\
\hline 9 & GShv- 273/07 x GAM- 173 & 49.00 & -3.92 & 0.00 & 16.17 & $-4.26 * *$ & $-5.33 * *$ \\
\hline 10 & GBhv- 282 x GBhv- 283 & 50.00 & -1.96 & 2.04 & 16.42 & $-4.20 * *$ & $-3.86 * *$ \\
\hline 11 & GBhv- 282 x GBhv- 286 & 51.00 & 0.00 & 4.08 & 16.84 & 0.54 & -1.41 \\
\hline 12 & GBhv- 282 x GBhv- 287 & 48.00 & $-5.88^{*}$ & -2.04 & 15.92 & $-4.21 * *$ & $-6.79 * *$ \\
\hline 13 & GBhv- 282 × 824 & 50.00 & -1.96 & 2.04 & 16.36 & $-3.08^{*}$ & $-4.22 * *$ \\
\hline 14 & GBhv- 282 x G 27 & 49.00 & $-12.50 * *$ & 0.00 & 17.29 & $3.53 *$ & 1.23 \\
\hline 15 & GBhv- 282 x GAM- 141 & 51.00 & 0.00 & 4.08 & 16.80 & $-3.89 * *$ & -1.64 \\
\hline 16 & GBhv- 282 x GAM- 165 & 49.00 & -3.92 & 0.00 & 16.30 & $-8.27 * *$ & $-4.57 * *$ \\
\hline 17 & GBhv- 282 x GAM- 173 & 50.00 & -1.96 & 2.04 & 17.52 & $3.73 * *$ & 2.58 \\
\hline 18 & GBhv- 283 x GBhv- 286 & 50.00 & 2.04 & 2.04 & 17.05 & -0.53 & -0.18 \\
\hline 19 & GBhv- 283 x GBhv- 287 & 48.00 & -2.04 & -2.04 & 17.05 & -0.53 & -0.18 \\
\hline 20 & GBhv- 283 x 824 & 49.00 & 0.00 & 0.00 & 15.99 & $-6.71 * *$ & $-6.38 * *$ \\
\hline 21 & GBhv- 283 x G 27 & 52.00 & $-7.14 * *$ & $6.12 *$ & 16.49 & $-3.79 * *$ & $-3.45^{*}$ \\
\hline 22 & GBhv- 283 x GAM- 141 & 49.00 & 0.00 & 0.00 & 16.69 & $-4.52 * *$ & -2.28 \\
\hline 23 & GBhv- 283 x GAM- 165 & 50.00 & 2.04 & 2.04 & 17.12 & $-3.66^{* *}$ & 0.23 \\
\hline 24 & GBhv- 283 x GAM- 173 & 48.00 & -2.04 & -2.04 & 17.29 & 0.88 & 1.23 \\
\hline 25 & GBhv- 286 x GBhv- 287 & 50.00 & 2.04 & 2.04 & 16.74 & -0.06 & -1.99 \\
\hline 26 & GBhv- 286 x 824 & 46.00 & $-6.12 *$ & $-6.12 *$ & 16.84 & -0.24 & -1.41 \\
\hline 27 & GBhv- 286 x G 27 & 52.00 & $-7.14 * *$ & $6.12 *$ & 17.22 & 2.81 & 0.82 \\
\hline 28 & GBhv- 286 x GAM- 141 & 48.00 & -2.04 & -2.04 & 17.39 & -0.51 & 1.81 \\
\hline 29 & GBhv- 286 x GAM- 165 & 49.00 & 0.00 & 0.00 & 16.11 & $-9.34 * *$ & $-5.68 * *$ \\
\hline 30 & GBhv- 286 x GAM- 173 & 49.00 & 0.00 & 0.00 & 16.63 & -1.54 & -2.63 \\
\hline 31 & GBhv- 287 x 824 & 51.00 & $6.25 *$ & 4.08 & 16.45 & -2.55 & $-3.69 * *$ \\
\hline 32 & GBhv- 287 x G 27 & 49.00 & $-12.50 * *$ & 0.00 & 17.47 & $4.61 * *$ & 2.28 \\
\hline 33 & GBhv- 287 x GAM- 141 & 48.00 & -2.04 & -2.04 & 16.76 & $-4.12 * *$ & -1.87 \\
\hline 34 & GBhv- 287 x GAM- 165 & 50.00 & 4.17 & 2.04 & 17.18 & $-3.32 *$ & 0.59 \\
\hline 35 & GBhv- 287 x GAM- 173 & 49.00 & 2.08 & 0.00 & 17.52 & $3.73 * *$ & 2.58 \\
\hline 36 & 824 x G 27 & 44.00 & $-21.43 * *$ & $-10.20 * *$ & 16.79 & -0.53 & -1.70 \\
\hline 37 & 824 x GAM- 141 & 51.00 & 4.08 & 4.08 & 16.93 & $-3.15^{*}$ & -0.88 \\
\hline 38 & 824 x GAM- 165 & 51.00 & $15.91 * *$ & 4.08 & 17.56 & -1.18 & $2.81 *$ \\
\hline 39 & 824 x GAM- 173 & 47.00 & -2.08 & -4.08 & 17.86 & $5.74 * *$ & $4.57 * *$ \\
\hline 40 & G 27 x GAM- 141 & 48.00 & $-14.29 * *$ & -2.04 & 17.25 & -1.32 & 1.00 \\
\hline 41 & G $27 \times$ GAM- 165 & 48.00 & $-14.29 * *$ & -2.04 & 16.86 & $-5.12 * *$ & -1.29 \\
\hline 42 & G $27 \times$ GAM- 173 & 50.00 & $-10.71 * *$ & 2.04 & 17.34 & 2.66 & 1.52 \\
\hline 43 & GAM- 141 x GAM- 165 & 47.00 & -4.08 & -4.08 & 16.63 & $-6.42 * *$ & -2.63 \\
\hline 44 & GAM- 141 x GAM- 173 & 46.00 & $-6.12 *$ & $-6.12^{*}$ & 17.58 & 0.57 & $2.93 *$ \\
\hline \multirow[t]{4}{*}{45} & GAM- 165 x GAM- 173 & 47.00 & -2.08 & -4.08 & 17.14 & $-3.55^{* *}$ & 0.35 \\
\hline & S.E. (d) \pm & & 1.48 & 1.48 & & 0.24 & 0.24 \\
\hline & CD 0.05 & & 2.93 & 2.93 & & 0.47 & 0.47 \\
\hline & CD 0.01 & & 3.87 & 3.87 & & 0.62 & 0.62 \\
\hline
\end{tabular}

*, ** Significant at $5 \%$ and $1 \%$ levels, respectively. 
(Standard Heterosis).

Estimates of heterosis

Expression of heterosis for yield and yield attributing characters: The seed cotton yield of diploid cotton is generally contributed by numbers of boll and boll weight in intraspecific hybrids and numbers of boll in interspecific hybrids (Singh et al., 1975 and Bhatade, 1983).

Mean range for no. of bolls/ plant varied from 22.41 (GBhv- 283 x GAM- 165) to 97.40 (GBhv- $282 \times$ G 27). Among 45 hybrids, 22 hybrids exhibited significant positive heterotic effect over their respective better parent. The cross GBhv- 287 x 824 was associated with maximum heterobeltiosis $(141.22 \%)$. Five hybrids demonstrated positive siginificant standard heterosis for no. of bolls/ plant. According to standard heterosis, promising cross combinations for no. of bolls/ plant were GBhv- 287 x 824 (41.59 \%), GBhv$282 \times$ GAM- $173(27.54 \%)$ and GBhv- 286 x G 27 $(21.05 \%)$. High heterotic expression for this particular trait would be useful for obtaining higher seed cotton yield. The significant heterosis for no. of bolls/plant (Gossypium spp.) which resulted in high yield was also recorded by Patil et al. (2009), Jyotiba et al. (2010), Patel et al. (2010), Sekhar babu et al. (2011) and Ashokkumar and Ravikesavan (2013).

In case of boll weight, mean performance of crosses showed that the cross GBhv- 283 x GAM- 165 (2.94 g) recorded the highest mean performance. The hybrid GShv- 273/07 x GBhv- 287 depicted the highest significant positive heterobeltiosis of 20.60 per cent. Fourteen hybrids noticed with positive significant standard heterosis, the highest value was observed for the cross GBhv- 283 x GAM- 165 (44.83\%). Other researchers, Patil et al. (2009), Jyotiba et al. (2010), Patel et al. (2010), Sekhar babu et al. (2011) and Tyagi et al. (2014) also found significant heterosis for Gossypium spp. boll weight.

The range of mean value among cross combination for seed cotton yield/ plant varied from $51.16 \mathrm{~g}$ (947.40 $\mathrm{kg} / \mathrm{ha})$ to $223.15 \mathrm{~g}$ (4132.40 kg/ ha). The extent of heterosis for seed cotton yield/ plant ranged from 43.77 to 155.60 per cent and -61.63 to 67.36 per cent over BP and SC, respectively. Among 45 hybrids, 17 showed significant positive heterosis over better parent while five hybrids indicated significant positive standard heterosis. Cross combinations, GBhv- 282 x G 27 (67.36\%), GBhv- 287 x 824 (58.14\%), GBhv- 282 x GAM- 173 (35.00\%), GBhv- 286 x G 27 (20.50\%) and GBhv- $283 \times 824(18.75 \%)$ were the top ranking heterotic crosses showed positive significant economic heterosis for seed cotton yield/ plant. Patil et al. (2009), Jyotiba et al. (2010), Patel et al. (2010), Saravanan and Koodalingam et al. (2011), Sekhar babu et al. (2011) and Ashokkumar and Ravikesavan (2013) also investigated significant heterosis for seed cotton yield/ plant.
Expression of heterosis for Fibre quality traits: In recent years, more emphasis is laid on quality traits from seed cotton yield in diploid cotton. For $2.5 \%$ span length, 13 and 27 hybrids exhibited significant heterosis in desired direction over better parent and standard check repectively. Among them the cross GBhv-286 x GBhv- 287 (17.58\%) was superior for heterobeltiosis and cross GBhv-286 x 824 (33.33\%) recorded maximum standard heterosis. The earlier researchers, Jyotiba et al. (2010), Sekhar babu et al. (2011), Rajamani et al. (2009) and Patil et al. (2012) also suggested improvement of fibre quality through heterosis as they recorded significant heterosis for different fibre quality traits in Gossypium spp..

As regards to the fibre strength, the heterobeltiosis ranged from -35.10 to 29.81 and cross 824 x GAM$141(29.81 \%)$ reported the maximum heterobeltiosis. Cross combinations GBhv-283 x 824 (34.64 \%), GBhv -286 x 824 (32.40\%), GShv-273/07 x 824 and GAM$141 \times$ GAM-173 (30.91\%) were on the top position for standard heterosis. The results corroborated the findings of Jyotiba et al. (2010) and Sekhar babu et al. (2011) for heterosis for the fibre strength trait in Gossypium spp.

For micronaire value, hybrid GBhv-286 x GAM-165 ($12.24 \%$ ) observed maximum heterobeltiosis while hybrid 824 x G 27 (-37.70\%) recorded maximum standard heterosis in desired direction. Majority of the cross combinations showed negative economic heterotic effect and decrease in micronaire value are an indication of fibre fineness. Kumar et al. (2003), Rajamani et al. (2009), Jyotiba et al. (2010), Sekhar babu et al. (2011) and Tuteja and Agrawal et al. (2013) but in contradiction of Patil et al. (2012) also reported the heterosis for fibre fineness in other cotton genotypes For fibre elongation percentage, the hybrids manifested significant heterobeltiosis, varied from -14.52 in $824 \times$ GAM- 141 and $824 \times$ GAM- 165 to 34.69 in GBhv- 282 x G 27 over better parental value in positive direction. Heterobeltiosis for fibre elongation percentage showed that the crosses GBhv-282 x G 27 (34.69 \%), GBhv-286 x G 27 (23.53\%) and GBhv-283 x GBhv-287 (18.52\%) were on top positions. In comparison with standard check, 18 hybrids exhibited positive standard heterosis and superior cross combinations over standard check were GBhv- 282 x G- 27 (17.86 $\%)$ and GShv- 273/07 x GAM- 165, GBhv- 283 x 824 and GBhv- 287 x 824 (16.07\%).

Fibre maturity is an important criterion for judging the suitability of lint for spinning. Formation of neps in the cotton while spinning is mainly due to the presence of immature fibres which accounts for breakage of yarn. Heterobeltiosis for fibre maturity coefficient showed that only two hybrids viz., GBhv-282 x G 27 (7.32\%) and GBhv-283 x G 27 (6.10\%) had significantly positive heterobeltiosis. None of the hybrids showed significantly positive standard heterosis. For short fibre 
index (SFI), hybrid GBhv-286 x GBhv-287 (-53.10\%) depicted maximum standard heterosis in desired direction. The occurance of heterosis for fibre maturity in Gossypium spp. was also reported by Kumar et al. (2003) and Patel et al. (2007a). For fibre uniformity ratio, only 2 and 4 hybrids reported positive heterosis over better parent and check respectively. Hybrids 824 x GAM-165 (15.91\%) and GBhv-287 x 824 (6.25\%) were observed highly significant for heterobeltiosis. Hybrids GShv-273/07 x GBhv-283 and GShv-273/07 x GBhv-287 (8.16 \%) were observed superior over check.

In case of oil content, among 45 hybrids only 5 and 3 hybrids exhibited the significant positive heterobeltiosis and standard heterosis respectively. In case of heterosis over better parent only five hybrids viz. $824 \mathrm{x}$ GAM- 173 (5.74\%), GBhv- 287 x G 27 (4.61\%), GBhv- 282 x GAM- 173, GBhv- 287 x GAM- 173 (3.73\%) and GBhv- 282 x G 27 (3.53\%). The range of standard heterosis varied from $-9.60 \%$ (GShv273/07 x GBhv- 286) to $4.57 \%$ (827 x GAM-173). Also, these results are in accordance with results of Ashokkumar and Ravikesavan (2013) in Gossypium hirsutum genotypes. Magnitude of heterosis for seed oil content was higher in intra and interspecific arboreum hybrids in comparison to intra herbaceaum hybrids.

\section{Conclusion}

Very less number of hybrids in diploid cotton are released in India, and hence the improvement in our native diploid cotton species which are sturdy in nature and provide better resistance against biotic and abiotic stress becomes imperative. Our study was aimed to exploit heterosis in desi cotton (Gossypium arboreum L. and $G$. herbaceum L) hybrids for yield, fibre quality as well as oil content. In the present experiment crosses GBhv- 282 x G 27, GBhv- 287 x 824, GBhv- 282 x GAM- 173, GBhv- 286 x G 27 and GBhv - 283 x 824 appeared to more promising for seed cotton yield/ plant. Boll number was the major yield contributing trait. Fibre quality as well as oil quality was higher in intra and interspecific arboreum hybrids.

\section{REFERENCES}

Ashokkumar K, Ravikesavan R (2013). Genetic variation and heterotic effects for seed oil, seed protein and yield attributing traits in upland cotton (Gossypium hirsutum L.). Afr. J. Biotechnol.12(33), 5183-5191.

Bhatade, S. S. 1983. Environmental influence on the magnitude of heterosis in G. arboreum L. Ind. J. Agric. Sci. 53 (8): 627-633.

Dock, J. W. and Moll, R. H. 1934. A new technique in cotton hybridization suggested changes in existing method of emasculating and bagging cotton flower. J. Heridity. 25: 201-204.

Ganapathy S, Nadarajan N (2008). Heterosis studies for oil content, seed cotton yield and other economic traits in cotton (G. hirsutum L.). Madras Agric. J. 95 (7-12):306310.

Jyotiba, S. S., Patil, B. R., Deshpande, S. K., Patil, S. S. and Patil, R. S. 2010. Heterosis Studies in GMS based diploid Cotton. Electronic J. Plant Breeding. 1(4): 685-688.

Khan N, Basal H, Hassan G (2010). Cottonseed oil and yield assessment via economic heterosis and heritability in intraspecific cotton populations. Afr. J. Biotechnol. 9 (44), 7418-7428.

Khan N, Basal H, Hassan G (2010). Cottonseed oil and yield assessment via economic heterosis and heritability in intraspecific cotton populations. Afr. J. Biotechnol. 9 (44), 7418-7428

Khan NU, Hassan G, Kumbhar MB, Kang S, Khan I, Parveen A, Aiman U, Saeed M (2007a). Heterosis and inbreeding depression and mean performance in segregating generations in upland cotton. Eur. J. Sci. Res. 17: $531-546$.

Khan NU, Hassan G, Kumbhar MB, Parveen A, Aiman U, Ahmad W,Shah SA, Ahmad S (2007b). Gene action of seed traits and its oil content in upland cotton $(G$. hirsutum). Sabrao J. Breed. Genet. 39:17-30.

Kumar, M., Chhabra, B.S. and Kumar, R. (2003). Heterosis for seed cotton yield and fibre quality characters in intra $G$. arboreum L. hybrids. J. Cotton Res. Dev. , 17(1): 27 - 29.

Meredith, W. R. and Bridge, R. R. 1972. Heterosis and gene action in cotton Gossypium hirsutum L. Crop Sci. 12: 304-310.

O.P.Tuteja and Manish Agrawal. 2013. Heterosis for Seed Cotton Yield and other Traits in GMS based Hybrids of American Cotton (Gossypium hirsutum). Cotton Res. J. 5(2) 131-141

Patel, J. P., Fougat, R. S., Jadeja, G. C., Patel, C. G. and Suthar, K. P. 2010. Heterosis study for yield and yield attributing characters in inter-specific Asiatic cotton hybrids. International J. Agricultural Sciences. 6(1): 7883.

Patel, K. G.; Patel, R. B.; Patel, M. I. and Kumar V. (2007a). Genetics of yield, fibre quality and their implication in breeding of interspecific cross derivatives of cotton. $J$. Cotton Res. Dev., 21 (2): $153-157$

Patil, S. S., Gavit, A. F., Magar, N. M. and Pawar, V. Y. 2009. Heterosis in hybrids of G. arboreum cotton. J. Cotton Res. Dev. 23(2): 209-212.

Patil, Sandip A., Naik, M. R., Pathak V.D. and Kumar V. (2012) - Heterosis for yield and fibre properties in upland cotton (Gossypium hirsutum L.) - J. Cotton Res. Dev., 26 (1): 26- 29.

Rai, B. 1978. Heterosis Breeding. Agro Biological Publications, New Delhi.

Rajamani, S.; Rao, C. M.; Naik, R. K.. (2009). Heterosis for yield and fibre properties in upland cotton, Gossypium hirsutum L. Journal of Cotton Research and Development. 23(1):. 43-45

Saravanan, S. and Koodalingam, K. 2011. Exploitation of hybrid vigour among interspecific crosses of Gossypium arboreum L. and Gossypium herbaceum L. Electronic J. Plant Breeding. 2(1): 143-146.

Sekhar Babu, G., Khadi, B. M., Mogali, S., Patil, R. S., Katageri, I. S., Nadaf, H. L. and Patil, B. C. (2011). Study of heterosis in genetic male sterility based diploid cotton hybrids for yield, yield component and fibre quality characters. Karnataka J. Agric. Sci. 24(2): 118-124. 
Sharma D, Pathak D, Atwal AK, Sangha MK (2009). Genetic variation for some chemical and biochemical characteristics in cotton seed oil. J. Cotton Res. Dev. 23 (1): 1 -7.

Singh, T. H., Khandola, H. S. and Negi, P. S., 1975. Hybrid vigour in intervarietal crosses of desi cotton (G. arboreum L.). J. Res., Punjab Agric. Univ., Ludhiana. 12: 218-221.

Tyagi, P., Bowman, D. T., Bourland, F. M., Edmisten, K., Campbell, B. T., Fraser, D. E., Wallace, T. and Kuraparthy, V. 2014. Components of hybrid vigor in upland cotton (Gossypium hirsutum L.) and their relationship with environment. Euphytica. 195: 117-127.
Wei XC, Li QZ, Pang JQ, Zhang J, Zhao JH, Wang LG (2002). Heterosis of pre-forest lint yield of hybrid between cultivars or lines within upland cotton $(G$. hirsutum L.). Cotton Sci. 14: 269-272.

Yuan YL, Zhang TZ, Guo WZ, Pan JJ, Kohel RJ (2002). Heterosis and gene action of boll weight and lint percentage in high quality fibre property varieties in upland cotton. Acta Agron. Sin. 28: 196-202.

Zhang ZS, Li XB, Liu DJ, Huang SL, Zhang FX (2002). Study on heterosis utilization of upland cotton $(G$. hirsutum L.) lines with high fibre quality. Cotton Sci. 14: 264-268. 